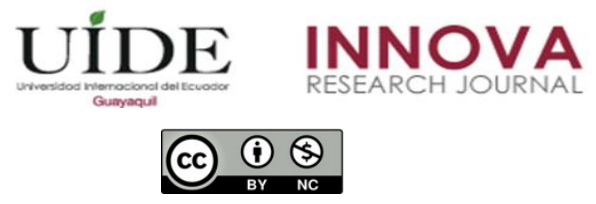

INNOVA Research Journal, ISSN 2477-9024

(Enero-Abril 2021). Vol. 6, No.1 pp. 145-161

DOI: https://doi.org/10.33890/innova.v6.n1.2021.1465

URL: http://revistas.uide.edu.ec/index.php/innova/index

Correo: innova@uide.edu.ec

\title{
La competitividad como factor de crecimiento para las organizaciones
}

\section{Competitiveness as a growth factor for organizations}

Gabriel Alejandro Díaz Muñoz

(iD) https://orcid.org/0000-0001-9891-5425

María Dolores Quintana Lombeida

(iD https://orcid.org/0000-0003-4049-0844

Daniel Gonzalo Fierro Mosquera

(D) https://orcid.org/0000-0003-3666-491X

Universidad Tecnológica Equinoccial, Ecuador

Autor para correspondencia: gabriel.diaz@ute.edu.ec; maria.quintana@ute.edu.ec;

daniel.fierro@ute.edu.ec

Fecha de recepción: 10 de julio de 2020 - Fecha de aceptación: 21 de octubre de 2020

\section{Resumen}

La competitividad es una de las condiciones más valoradas y perseguidas por los directivos organizacionales quienes conscientes de la creciente demanda y expectativas de la clientela, cada vez más exigente y compleja, intentan sacar sus mejores armas de batalla para ocupar una posición privilegiada en el mercado y asegurar su permanencia a mediano y largo plazo. Esta investigación tiene el objetivo de brindar al lector un documento amigable y de fácil comprensión que permita entender la importancia que tiene la competitividad como factor de crecimiento para las organizaciones. Para ello, el documento inicia con una revisión bibliográfica cimentada en la teoría y perspectiva que diferentes autores tienen a acerca de la materia en cuestión y en lo posterior, mediante una metodología descriptiva, correlacional y cualitativa se realiza un análisis de las posturas de los autores citados a lo largo del texto relacionando temas como la competitividad y la estrategia empresarial, el liderazgo a través de la competitividad, la productividad como elemento determinante, factores que merman la competitividad, la globalización y la importancia del capital humano. En base al análisis realizado, se concluye señalando que la competitividad ha dejado de ser un concepto estático centrado en aspectos de naturaleza financiera, ahora se valora mucho el vínculo entre los activos de naturaleza intangible, calidad del recurso humano y motivación del personal, por ende, el liderazgo de la cúpula empresarial es un factor determinante y una de las razones por las que algunas organizaciones no pueden alcanzar los niveles de competitividad requeridos o deseables.

Palabras claves: competitividad; empresa; crecimiento organizacional; desarrollo, eficiencia.

\begin{abstract}
Competitiveness is one of the conditions most valued and pursued by organizational managers who, aware of the growing demand and expectations of the increasingly demanding and complex clientele, try to draw their best battle weapons to occupy a privileged position in the market and
\end{abstract}


ensure its permanence in the medium and long term. This research aims to provide the reader with a friendly and easy-to-understand document that allows understanding the importance of competitiveness as a growth factor for organizations. For this, the document begins with a bibliographic review based on the theory and perspective that different authors have about the matter in question and subsequently, through a descriptive, correlational and qualitative methodology, an analysis of the authors' positions is carried out cited throughout the text relating issues such as competitiveness and business strategy, leadership through competitiveness, productivity as a determining element, factors that reduce competitiveness, globalization and the importance of human capital. Based on the analysis carried out, it is concluded by pointing out that competitiveness is no longer a static concept focused on aspects of a financial nature, now the link between assets of an intangible nature, quality of human resources and motivation of staff is highly valued, therefore, leadership from the corporate leadership is a determining factor and one of the reasons why some organizations cannot achieve the required or desirable levels of competitiveness.

Keywords: competitiveness; company; organizational growth; development; efficiency.

\section{Introducción}

La competitividad constituye una herramienta estratégica que, con el pasar de los años, ha ido cobrando mayor importancia no solo entre los académicos sino también entre las empresas, pues diferentes aristas referentes a este concepto pueden ser analizadas para entender por qué es trascendental para las organizaciones adoptar la competitividad como una filosofía de vida.

Este documento no persigue como finalidad otra cosa que, analizar la competitividad como factor de crecimiento y desarrollo sostenido en el tiempo para las organizaciones. La primera parte de esta investigación comienza definiendo la competitividad desde la perspectiva de diferentes autores y cómo se consigue el liderazgo en las organizaciones por medio de esta herramienta estratégica; posteriormente y mediante una metodología descriptiva, correlacional y cualitativa, se analiza cómo influye la productividad en los niveles competitivos que puede alcanzar la industria y los factores que la pueden afectar valiéndose para ello de cuatro principales criterios de análisis: el liderazgo, la estrategia empresarial, la productividad y la competitividad. Para concluir, se relaciona la globalización como oportunidad para explorar nuevos mercados y el talento humano como elementos claves en las organizaciones.

La competitividad no es un concepto nuevo. Según Galindo (2008), en su libro Diccionario de Economía Aplicada: Política económica, economía mundial y estructura económica, este término proviene desde hace tres siglos aproximadamente cuando Adam Smith desarrolló la teoría de la ventaja absoluta relacionándola con el comercio internacional y los beneficios para todos los actores partícipes del proceso en su obra La Riqueza de las Naciones publicada en 1776.

Posteriormente, David Ricardo hizo aportes importantes referentes a este tema a través de su obra Principios de economía en 1817 mediante la teoría de la ventaja comparativa, la cual señalaba que un país lleva la delantera en la generación de un bien cuando lo puede producir a un coste relativo menor que otros países. Por su parte, Porter (2015) asegura que la competitividad 
depende de la capacidad de la industria para innovar y mejorar; las compañías a su vez ganan ventajas sobre los mejores competidores del mundo debido a la presión y al reto.

Hoy en día, se encuentran con frecuencia publicaciones e información referente a este término en el entorno empresarial debido a la importancia que conlleva para cualquier organización alcanzar niveles aceptables de competitividad y de esa manera, asegurar su permanencia en el mercado. En las páginas posteriores de este documento, se hará referencia a la competitividad que debe ser entendida no como un fin, sino como una herramienta a través de la cual se puede lograr crecimiento, diferenciación y liderazgo.

\section{Desarrollo}

La competitividad es un tópico que desde hace décadas despierta el interés y curiosidad en los líderes y gerentes de empresa puesto que su acertada gestión se mide, en gran parte, por la capacidad de administrar eficientemente los recursos disponibles en la organización y que esto a su vez, redunde en el incremento de la productividad. Para comprender con más claridad la temática propuesta en este documento, a continuación, se abordarán algunas conceptualizaciones y aportes realizados por diferentes autores.

\section{La competitividad}

Cordero, Chavarría, Echeverri y Sepúlveda (2003), se refieren a ella como la capacidad de mantener y ampliar la participación de las empresas en los mercados locales e internacionales de una manera lucrativa que permita su crecimiento. Según Fernández, Montes y Vázquez (1997) la competitividad de las empresas es un factor clave del crecimiento económico de una nación en un entorno mundial cada vez más abierto y dinámico.

La competitividad empresarial puede ser comprendida de distintas maneras; pero, en general, hace referencia a un conjunto de herramientas que, combinadas entre sí, facilitan liderar el mercado y vencer a los competidores del medio. Su importancia reside según Porter (2017) en el incremento de la productividad mediante el uso de recursos, pues saber administrarlos de manera óptima, permite además, estar siempre prestos a responder con rapidez a los requerimientos del mercado.

La competitividad se presenta como producto de la rivalidad constante entre las empresas del medio y se logra mediante un proceso de gestión dinámico entre la industria y sus grupos de interés - clientes, proveedores, directivos, acreedores, competidores del mercado — a fin de mostrarse ante la sociedad como un ente capaz de satisfacer las necesidades de sus consumidores mejor que la competencia.

Según Rubio y Baz (2015), la competitividad de cada empresa depende de sus propias estructuras internas, es decir, de su organización y capacidad de producir de una manera tal que pueda elevar sus ventas y ganarles a sus competidores en distintos ámbitos. Esto evidencia que para mejorar la competitividad de la industria, el primer paso empieza por sistematizar sus procesos operativos y definir la capacidad de producción, infraestructura y todos aquellos 
elementos que forman parte de la estructura interna, puesto que una vez identificados, es más fácil de diseñar e implementar políticas institucionales que favorezcan el entorno laboral a fin de permitir a la organización, ser cada vez más competitiva.

\section{La competitividad y la estrategia empresarial}

La competitividad comprende una de las fuerzas más poderosas presentes en la sociedad puesto que permite avanzar en términos de crecimiento socioeconómico y actualmente es un fenómeno a nivel mundial que incluye a empresas, países. La sociedad moderna en la que se vive actualmente, es protagonista de la alta competitividad que se ha intensificado en todas las áreas del conocimiento. Esto como es lógico, conlleva a que las organizaciones se vean en la necesidad de precisar estrategias que permitan ofrecer valor agregado a los productos o servicios que entregan al mercado y, de esta manera, se puedan satisfacer las necesidades de sus clientes más rápido que la competencia.

La estrategia competitiva según Porter (1985), implica posicionar una empresa para maximizar el valor de las capacidades que la distinguen de sus competidores; a la vez, el objetivo de cualquier estrategia genérica es crear valor para los compradores.

La creación de una estrategia empresarial implica mucho esfuerzo y tener los objetivos bien definidos, es indispensable para sobresalir en el mundo de los negocios pues, sin importar cuan claras estén las metas, si es que no se cuenta con las herramientas necesarias, difícilmente se lograrán obtener los resultados esperados, peor aún ventajas competitivas que permitan sobresalir entre las empresas del medio.

La creación de una posición competitiva ventajosa como resultante de una buena estrategia empresarial que según Carrión (2007), se relaciona con la toma de decisiones y persigue conseguir posiciones competitivas superiores respecto a los competidores, intentando generar capacidades distintivas.

Es importante tener en cuenta que los mercados y las realidades del entorno no son siempre iguales, ya que se encuentran en un proceso de evolución y transformación continua; por ende, pueden cambiar las condiciones que en un principio permitieron a una empresa contar con una ventaja competitiva y esto requiere que los planes y políticas deben estar diseñados para adaptarse a las realidades que atraviesa la organización en distintas etapas de su vida empresarial.

La competitividad puede representar el factor determinante para el fracaso o éxito de las organizaciones y además, para mantenerse en el mercado es importante vencer a la competencia mediante la creación de una ventaja competitiva sostenible en el tiempo. Este concepto desarrollado por Porter (1980) permite entender la importancia del desarrollo de una o varias estrategias que necesariamente deberán tener comportamientos determinados.

Si por ejemplo, el objetivo es competir en el mercado por precios bajos, las políticas institucionales deben estar encaminadas a mitigar desperdicios, seleccionar estrictamente los proveedores y manejar costos de producción de forma más eficiente que la competencia. Si por 
el contrario, la meta es competir por diferenciación, la calidad de los productos y la atención al cliente deben estar direccionadas a la búsqueda de la excelencia empezando por la calidad de la materia prima, capacitación al personal en atención al cliente, control estricto y estándares de calidad en los productos, procesos de mejora continua, etc., aunque todo esto implique un aparente costo de producción más elevado, pues todo tiene que estar alineado a la estrategia definida por la gerencia.

La estrategia es un término que se ha vuelto cada vez más importante con el paso de los años; tanto es así, que expertos han venido realizando aportes importantes vinculando este concepto como elemento determinante en el mundo de los negocios empezando con Miles y Snow (1978), Porter (1980), Miller (1987), Mitzberg (1988) y, por último Kotler (1992).

Kaplan y Norton (2014) también han realizado aportes en relación a la estrategia empresarial asegurando que no es únicamente un proceso de gestión o, al menos, no debería considerarse como tal puesto que comprende un proceso continuo y lógico que implica el trabajo dinámico de los empleados.

Si bien es cierto, las empresas pueden parecer muy diferentes entre sí en función de su actividad económica, las teorías propuestas por los autores mencionados en el apartado anterior, ayudan a comprender el comportamiento de las mismas frente al mercado y también al por qué de la utilización de determinadas estrategias para difundir su propuesta, dar a conocer sus productos y acercar el bien o servicio al consumidor final de forma diferente a la que acostumbran los competidores pese a que dicho producto, aparentemente tenga similitud o inclusive, parezca igual al que producen otras empresas.

Autores de importante trayectoria académica como Michael Porter, un reconocido académico y considerado por muchos como el padre del marketing moderno, quien a su haber tiene un sinnúmero de publicaciones, ha definido tres estrategias que sirven como punto de partida adecuado para el pensamiento estratégico: liderazgo en costos, diferenciación y enfoque.

Liderazgo en costos: La empresa se esfuerza para obtener los costos de producción y distribución más bajos y así vender a precios más bajos que sus competidores y conseguir una mayor participación en el mercado. El problema que plantea esta estrategia es que siempre surgirán otras empresas con precios aún más bajos, lo que puede perjudicar a la empresa que apueste todo su futuro a los costos.

Diferenciación: La empresa se concentra en alcanzar mejores resultados con base en alguna ventaja importante que valora la mayor parte del mercado. La empresa debe concentrarse en aquellas fortalezas que contribuyan a la diferenciación.

Enfoque: La empresa se concentra en uno o más segmentos estrechos del mercado, la empresa llega a conocer estos segmentos en profundidad, y busca ser líder en costos o diferenciación dentro del segmento meta". (Kotler y Lane, 2006, p. 56).

Cada organización debe ser capaz de identificar los elementos que van a formar parte de la estrategia, que pueden afectar el cumplimiento de sus objetivos, y en función de ello, 
determinar los talentos, el recurso económico y tecnológico necesarios para alcanzar dichas metas.

En la figura 1, se muestra la denominada "Rueda de la estrategia competitiva", considerada un instrumento para integrar los aspectos esenciales de la estrategia. El centro describe las metas de la organización, mientras que los rayos de la rueda representan las políticas operáticas por medio de las cuales se pretende alcanzar las metas, tal como se muestra a continuación:

\section{Figura 1}

La rueda de la estrategia competitiva

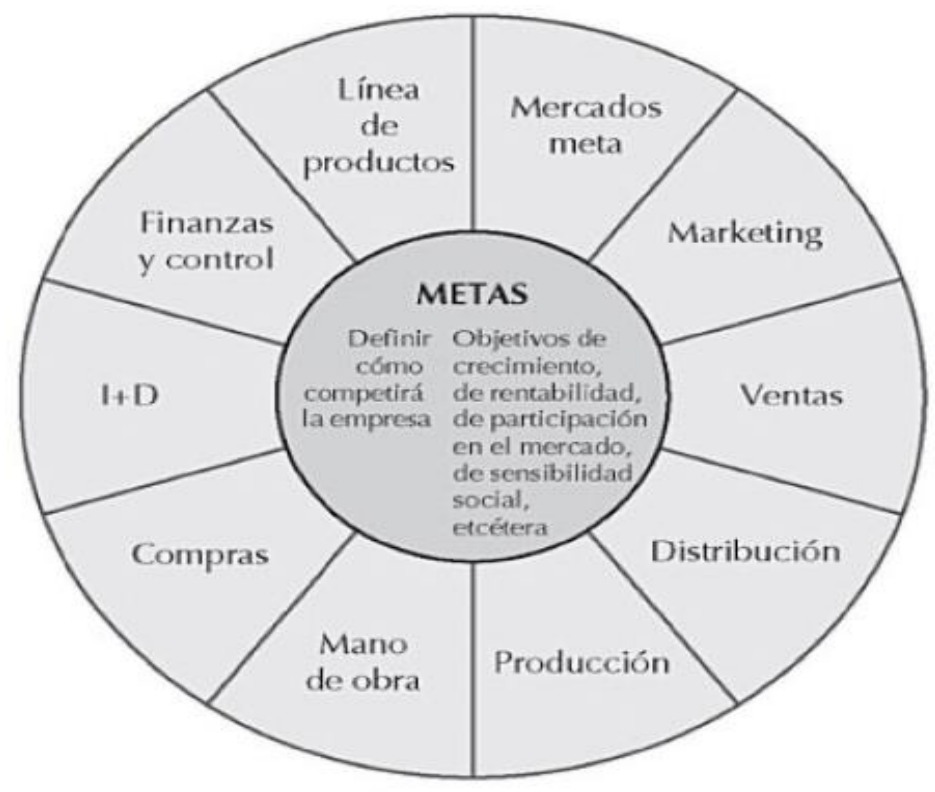

Fuente: Porter (2015).

La grafica anterior, permite visualizar todos aquellos elementos que forman parte de la estrategia la misma que gestionada de manera adecuada, permite la obtención de una característica percibida como única e inigualable por el mercado; no obstante, Corredor (2017, p. 82) sostiene que "una ventaja resultará efímera puesto que más pronto que tarde será copiada y mejorada por sus competidores".

Ante tal situación, es necesario pensar en todos los elementos que comprenden la estrategia empresarial, analizar aquellos que pueden ser copiados y fácilmente imitados por la competencia, que ya se visualizan actualmente en los productos o servicios que se encuentran en el mercado y de manera, atacar otros frentes seleccionando como factores de diferenciación, aquellos que permiten agregar valor al producto y que serán bien vistos por el consumidor con el objetivo de ocupar una posición privilegiada en su mente. 


\section{Liderazgo empresarial a través de la competitividad}

Existe literatura extensa en relación al liderazgo por lo que emitir una definición específica sería bastante limitado, así lo sostiene Sánchez (2017), quien define como líder a alguien cuyos colaboradores lo reconocen como tal.

Maxwell (2004) por su parte, se refiere al líder genuino como aquella persona que se reconoce porque de alguna manera, el personal a su cargo demuestra consecuentemente, tener un rendimiento superior al promedio.

El liderazgo responde a una serie de características en común que tienen todos los directivos empresariales respecto al deseo y la ambición por influir en las actitudes de otras personas y conseguir de esa manera resultados positivos para la industria en el mediano y largo plazo.

Existen algunas cualidades propias de los gerentes tales como el carisma, sensibilidad, seguridad en sí mismos, capacidad de motivación, entre otros, que comúnmente se cree que son cualidades innatas de los líderes; no obstante, Abarca (2013), señala que el liderazgo se basa en lo que los demás ven, no en una cualidad innata por cuanto implica un verdadero desafío, encontrar un equilibrio entre la personalidad propia de cada individuo y la capacidad de influir en la personalidad de otros.

Las características descritas anteriormente y combinadas en conjunto, proveen de confianza al equipo de trabajo y hacen posible dirigir a la empresa por el sendero que conduce a la consecución de los objetivos institucionales; sin embargo, "en el mundo actual, es claro que el liderazgo importa y las empresas sobresalientes así como los individuos de éxito, exhiben profundidad en el liderazgo y son capaces de emplear los atributos de liderazgo de manera apropiada y con un propósito". (PSICORE, 2014, p. 4).

La motivación de los empleados comprende el motor impulsor de la mejora continua, el mayor ente generador de valor agregado para las organizaciones modernas y representa uno de los tantos beneficios que conlleva un liderazgo bien entendido. Este término, a través de los años ha sido definido por distintas maneras por expertos, así por ejemplo, Chiavenato (1999), se refiere al liderazgo como la influencia interpersonal ejercida en una situación, orientada a la consecución de uno o de diversos objetivos mediante el proceso de comunicación humana.

La fluidez comunicacional en la empresa es parte esencial de la estrategia y el diseño de la mima, debe ser adaptada a las necesidades del consumidor que va evolucionando constantemente, las expectativas son cada vez más altas y sus requerimientos suelen ser muy distintos de lo que se mostraban años o meses atrás.

Esto implica que la organización y su naturaleza dinámica y adaptativa, debe estar en constante proceso de transformación y cambio pues, la estaticidad y falta de innovación son dos de los principales problemas que generan clientes insatisfechos y decremento en las ventas. Estas situaciones, aparte de poner en riesgo la supervivencia de la organización, generan otras tantas consecuencias negativas como deterioro de la imagen institucional y desmotivación en el 
personal que al percatarse de una baja en el nivel ventas y producción de la industria, tiende a bajar su rendimiento y como resultado de ello, los niveles de competitividad decaen de manera importante.

El liderazgo, por tanto, debe estar enfocado en algunos elementos que tienen relación a la actitud del trabajador frente al entorno laboral tales como el sentido de identidad con la organización, la motivación y la mejora del desempeño en el puesto de trabajo.

Este último, es uno de los factores más importantes pues tiene repercusión directa en el resultado de los productos o servicios que la empresa entrega a su clientes, por ende, la relación entre la compañía y los stakeholders debe gestionarse de forma permanente en un marco de consideración, respeto y crecimiento mutuo que permita el desarrollo económico de la industria pero también, a cada integrante de la organización, la oportunidad de mejorar en el ámbito personal, profesional y económico de manera que el beneficio sea bidireccional.

Por paradójico que parezca, la causa más frecuente para que gran parte de empresas no logren alcanzar el éxito, pese a contar con buena infraestructura y talento humano, es precisamente la carencia de liderazgo por parte de la cúpula organizacional, por tanto, definir las persona con las habilidades necesarias y capacidades de influir positivamente en el equipo de trabajo es un elemento absolutamente importante en materia de liderazgo y mejora en la competitividad empresarial.

\section{La productividad como factor determinante de la competitividad}

Olavarrieta de la Torre (1999), define la productividad como la relación entre producción e insumo, la relación entre lo que sale y lo que entra, o también la relación entre lo que se obtiene y los recursos usados para producirlo.

Es común relacionar el término competitividad con productividad; este vínculo, actualmente, representa un principio en el mundo de los negocios y una filosofía de vida para las organizaciones modernas puesto que "en la medida en que una empresa es más productiva, su capacidad de competir será mayor y por ello, hablar de competitividad implica necesariamente hablar de productividad" (Rubio y Baz, 2015, p. 7).

Como se puede comprender, productividad y competitividad son dos conceptos afines pero de diferente significado. Cuando se intenta definir la competitividad es común relacionar un conjunto de características que tiene una empresa las cuales ayudan a sobresalir frente a sus competidores; es decir, la competitividad refiere en mayor medida a la calidad del producto o servicio entregado al mercado y su aceptación por parte de los consumidores. La productividad, por su parte, implica el aprovechamiento máximo de recursos con los que cuenta la industria en pro de obtener el mayor provecho en términos de cantidad o unidades de productos fabricados.

Generalmente, se suelen asociar los términos productividad y competitividad pero no siempre queda clara la diferencia entre ambos vocablos. Huertas y Dominguez (2008), hacen referencia a estos conceptos al mencionar que la competitividad tiene relación a las posiciones 
que ocupa un producto o empresa en el mercado frente a otros productos o empresas y la productividad comprende el conjunto de factores que contribuyen a la posición competitiva de dichos productos fabricados por un país o industria.

Se dice que una empresa es productiva siempre que esté en la capacidad de mejorar el rendimiento, aprovechar al máximo sus recursos disponibles y pueda adaptarse al cambio continuo y necesidades latentes del público de manera que puedan producir más y mejor que la competencia pero utilizando la menor cantidad de recursos.

En este contexto, una de las equivocaciones más frecuentes es deducir que si una empresa tiene una gran capacidad de producción es competitiva automáticamente, lo cual es totalmente falso. Por el contrario, ocurre que si una empresa produce bienes o servicios de calidad excepcional pero en una cantidad ínfima, esto se traduce en ingresos insuficientes lo cual genera como consecuencia una rentabilidad económica irrisoria para la industria.

Cuando se habla de productividad y competitividad, las industrias generalmente concentran todas sus armas de batalla en ser productivas, pero se descuidan en gran medida los índices de competitividad. Esto se genera cuando se olvida manejar de manera adecuada los procesos productivos que, según Fúquene (2007), están relacionados con un conjunto de operaciones y actividades que, ejecutadas de manera sistemática y ordenada, permiten crear valor mediante la transformación de insumos o materias primas en un producto o servicio.

La planeación de procesos productivos permite llevar a cabo adecuadamente las fases de ejecución, control y determinar el personal operativo y administrativo necesario para realizar dichos procedimientos a través de los cuales, las industrias acercan los productos o servicios al consumidor toda vez que las características de los mismos, se encuentra al nivel o por encima de los competidores por lo que gestionar aspectos en materia de costos, plazos de entrega, calidad y flexibilidad es indispensable. (Cuatrecasas, 2016).

\section{Factores que afectan la competitividad de las empresas}

Hernández (2000) sostiene que la competitividad se mide mediante diferentes indicadores tales como las condiciones físicas de la planta, la organización de los procesos productivos, sistemas de incentivos y los de pagos por resultados son elementos que influyen preponderantemente; sin embargo, Alonso (2010) en contraposición a esta postura, afirma la competitividad es un concepto relativo y los elementos que la determinan y pueden afectar su medición, varían en función de las variables que se tomen como referencia.

El entorno corporativo constituye un factor relevante y muy amplio que comprende un conjunto de fuerzas sociales y económicas, mismas que deben analizarse en vista de que de alguna $u$ otra manera afectan a todas las empresas y por ende, no puede valorarse de la misma forma a todas las industrias pues, el mercado y las cualidades de sus productos o servicios exigen considerar como parámetros de medición a aquellos elementos que forman parte de la estrategia y que por ende, van a definir el comportamiento entre unas y otras. 
Existen factores que pueden implicar un peligro para la competitividad de las empresas y esto requiere que los planes de acción y procesos de gestión, estén encaminados a mitigar los riesgos y amenazas que pueden afectar dramáticamente la disponibilidad de suministros, escasez o demoras, y otros acontecimientos, los cuales según Rojas, Chavarría y Sepúlveda (1999), suponen un costo a corto plazo en las ventas y daño en la satisfacción del cliente a largo plazo.

Son muchos los elementos que pueden desencadenar en el detrimento de la competitividad empresarial, algunos pueden ser factores externos ajenos al control de la empresa pero otros que si pueden gestionarse en el interior de la misma. Los más comunes son errores de manufactura que son detectados al final de la cadena de producción generando como consecuencia, un reprocesamiento con los consecuentes costes de materia prima, mano de obra y tiempo de producción que ello implica. Este tipo de situaciones se originan por causa de equivocaciones del personal operativo y generalmente ocurren falta de comunicación, poco o nulo orden en la ejecución de los procesos productivos y falta de control que incluyen estándares de calidad en la materia prima.

Resulta entonces de vital importancia, considerar este tipo de situaciones y manejar de manera eficiente la gestión de procesos productivos en pro de mejorar sus niveles de competitividad y de esa manera, intentar asegurar la supervivencia y la permanencia en el mercado que en base a la postura de Anderson (1982), depende en gran medida de que las necesidades reales de los clientes puedan ser satisfechas oportunamente partiendo de un análisis de las oportunidades que se visualizan en el mercado a fin de crear nuevos productos, mejorar los ya existentes o identificar nuevas necesidades que aún no han sido satisfechas.

Estudiar el entorno competitivo puede afectar la competitividad de la industria por lo que realizar un estudio holístico antes de definir la estrategia y los planes de acción a seguir se vuelve absolutamente necesario. Porter (2017), señala cinco fuerzas competitivas que resultan importantes de analizar a fin de comprender en que forma pueden coadyuvar o mermar la competitividad de la organización y cómo se relacionan entre sí cada una de ellas, tal como se muestra en la figura 2: 


\section{Figura 2}

Las 5 fuerzas que moldean la competencia en un sector

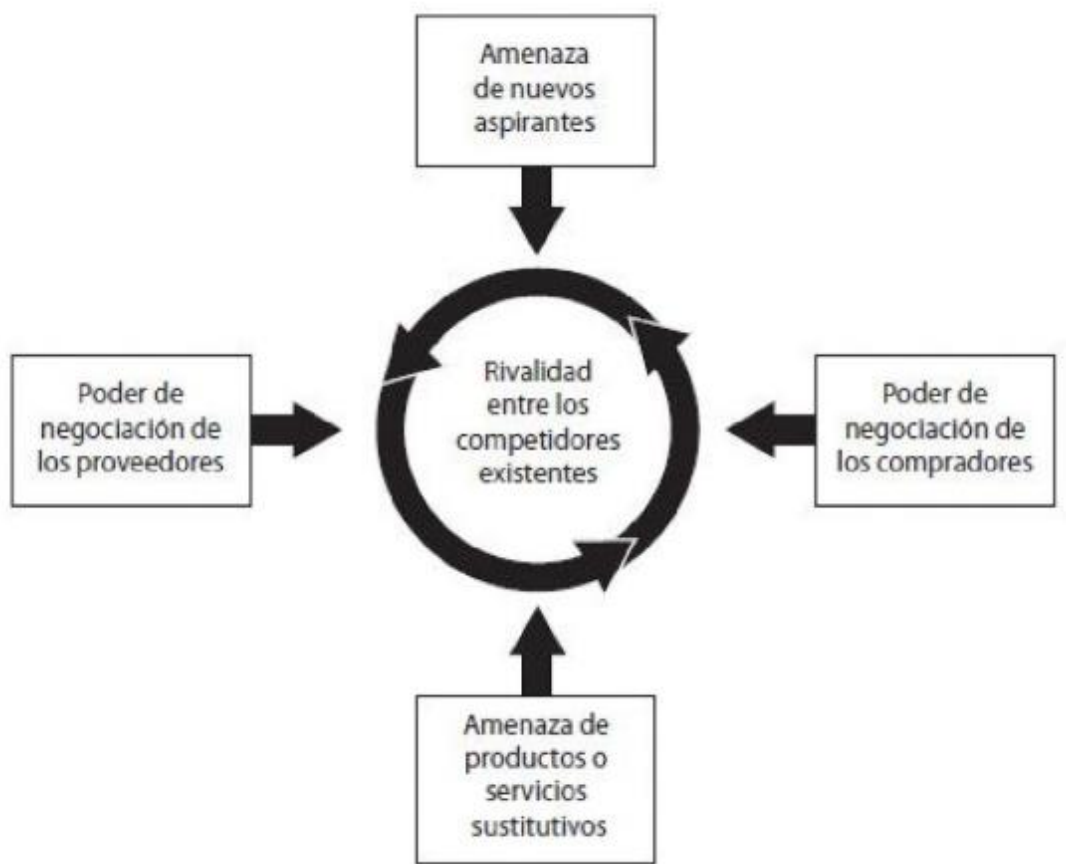

Fuente: Porter (2017).

Esta teoría propuesta por el reconocido académico Michael Porter, resulta de gran valía en el entorno empresarial y comprende un elemento que no puede pasar desapercibido ni darle poca importancia al momento de definir las reglas del juego y las oportunidades de penetración en el mercado que tiene la industria.

La intensidad de la competencia en el mundo de los negocios no es casualidad ni un asunto del azar, pues la naturaleza del sector industrial presenta una estructura económica basada en el comportamiento de los competidores y, a partir de ello, es que se puede determinar las fortalezas de la industria, sus puntos débiles que una vez identificados, permiten diseñar la estrategia con la que se pretende ganar la pugna por acaparar el mercado de mejor la manera.

Las empresas competitivas tienen la particularidad de ser entes capaces de operar y lograr un crecimiento económico sostenido a través del tiempo, esto se logra cuando las estrategias están direccionadas y enfocadas hacia las necesidades insatisfechas del mercado creando de esa manera, valor para sus accionistas en un entorno rodeado de competidores creativos y capaces de formular nuevas propuestas que satisfagan las expectativas de un público cada vez más exigente. 


\section{La globalización y la competitividad}

Hoy en día, el entorno empresarial se torna cada vez más competitivo, la competencia entre las empresas es más agresiva y esto es el resultado de la economía globalizada y facilidad de acceso a información de productos y servicios mediante las facilidades y rapidez que brindan las herramientas y medios digitales.

La competitividad no es un tema nuevo; su inicio se remonta a los orígenes de la humanidad, cuando el ser humano intentaba producir algo diferente y mejor que su competidor valiéndose para ello de herramientas tales como la innovación y la creatividad con el fin de ganar la aceptación y promover el deseo de compra por parte de los consumidores de la época.

Las organizaciones tienen actualmente una presión muy grande por mantenerse en un mercado altamente competitivo, su éxito se ve ligado a un alto sentido de productividad, eficiencia y eficacia en los procesos operativos siendo los sistemas de distribución y comercialización de sus productos, uno de los elementos determinantes para la expansión hacia nuevos mercados y nuevos clientes.

No depender única y exclusivamente de los clientes locales se vuelve cada vez más importante en la era modera, antiguamente, las industrias atendían la demanda insatisfecha de los consumidores mediante productos y servicios que se ofertaban en el mercado nacional, ahora la empresa está en capacidad de operar a nivel mundial y desarrolla sus estrategias de comercialización para adaptarse a mercados internacionales en procura de abrir sus horizontes y continuar creciendo social e económicamente hablando, todo ello producto de las ventajas y facilidades que brinda la globalización.

Bueno y Morcillo (1994, p. 199) describen este término como "el resultado de la integración de la economía mundial que ha puesto al alcance de las manos factores, recursos, conocimientos, consumidores y otros elementos que contribuyen al desarrollo de las empresas".

La economía de los mercados ha sufrido una transformación importante por causa de la globalización, por medio de ella, los mercados son abiertos y los productos o servicios que ofertan las empresas pueden ser accesibles a consumidores de diferentes territorios al lugar donde opera la empresa. Esta situación redunda en la necesidad de hacer las cosas de mejor manera tratando de ser competitivos para lograr aceptación y adhesión de los compradores sin importar su lugar de ubicación.

Actualmente, no existe empresa grande o pequeña que se encuentre a salvo de la globalización, pues este es un fenómeno creciente a nivel mundial y se presenta como consecuencia de la integración de la economía global que ha permitido acercar materia prima, insumos para la producción y comercialización de bienes o servicios, nuevos saberes que contribuyen a la innovación y mejora de los productos existentes, compradores con distintos gustos o preferencias y demás elementos que coadyuvan al crecimiento y desarrollo de la industria. 


\section{El capital humano como elemento clave}

Todas las empresas en la actualidad, crean un valor sostenible mediante la potenciación de sus activos intangibles: capital humano, bases de datos y sistemas de información, procesos de alta calidad, relaciones con los clientes y marcas, capacidad de innovación, cultura. (Kaplan y Norton, 2014).

El entorno empresarial que envuelve a las organizaciones se caracteriza por ser más competitivo que antes, esto implica que las empresas deben estar mejor preparadas y capacitadas que lo que ocurría en décadas pasadas y además, que se gestionen los activos intangibles como elemento clave y factor imprescindible de supervivencia en tiempos de crisis.

En un principio, era común asociar la competitividad empresarial a factores tangibles, entendiendo como tal a aquellos activos de naturaleza financiera que contribuyen a la continuidad de los procesos productivos y sostenibilidad de la empresa. Con el pasar de los años, este paradigma ha ido cambiando de forma importante al grado de vincular la competitividad de las empresas a factores que se caracterizan por tener una identidad inmaterial siendo el capital humano uno de los recursos que cumple con esta particularidad y que ayuda a desarrollar ventaja competitiva cuando es correctamente gestionado.

Según Blanco (2015), las personas podrán ser fuente de ventaja competitiva para las organizaciones en la medida en que sean únicas y valiosas, y las empresas competidoras no puedan hacerse con otras semejantes.

El capital humano en una organización comprende un recurso valioso dado las particularidades y características individuales y propias de cada persona, pero desafortunadamente durante años se pensó que los departamentos más importantes de una organización eran las áreas de producción y finanzas.

Con el pasar del tiempo, el capital humano ha pasado de ser el recurso con menor importancia a convertirse en la principal fuente de ventajas competitivas acorde a lo expuesto por López y Grandío (2005, p.59), quienes señalan que "el conjunto de habilidades, conocimientos y competencias de las personas que trabajan en la empresa, es una fuente incuestionable de ventajas competitivas a largo plazo".

Las ventajas competitivas permiten lograr diferenciación y posicionamiento en el mercado llegando así a generar réditos económicos que es el fin que persigue toda organización. El poder económico de las empresas es muchas veces lo que atrae a los mejores talentos y despierta la atención en otros tantos aspirantes del mercado laboral, a ocupar un puesto en la industria.

Actualmente, la competitividad comprende un tópico que se encuentra latente en los sectores de actividad económica, al igual que la competitividad entre las empresas, existe también competitividad en el talento humano pues hoy en día existen profesionales cada vez más preparados y creativos que son seleccionados por las empresas cuyos productos o servicios requieren un mayor intelecto y mejor desempeño en el puesto de trabajo. 
Ya no es suficiente contar con infraestructura y equipamiento necesario, resulta fundamental para cualquier industria contar con personal calificado, motivado, con alto sentido de pertenencia institucional y sobre todo, cuyo rendimiento sea óptimo en procura de que los resultados que las empresa entrega a sus públicos, cumplan e inclusive superen sus expectativas por lo que la gestión del talento humano, comprende un tópico de suma importancia para la competitividad organizacional y es claro que "solo las empresas que gestionen de forma eficiente sus activos intangibles podrán sobrevivir a largo plazo al aumentar el valor para sus accionistas". (Barceló et al., 2001, p. 157).

Los mercados han ido evolucionando y con ello, la manera en que las empresas atienden las necesidades de sus clientes. En este contexto, existen empresas disímiles pues hay algunas obsoletas, carentes de dinamismo, estáticas y con un sistema burocrático que les impide llegar a ser competitivas y otras que, por el contrario, tienen una filosofía de vida empresarial innovadora, de aprendizaje y capacitación continua que facilita su progreso y sostenibilidad al mediano y largo plazo. Este tipo de situaciones, se derivan en primer lugar del liderazgo por parte de la gerencia pero es indispensable también, contar con un equipo de trabajo que este presto a lograr los mejores resultados en pro de entregar al mercado productos o servicios con valor agregado.

Kaplan y Norton (2014), señalan que todas las empresas de hoy en día crean un valor sostenible mediante la potenciación de sus activos intangibles como el capital humano, relaciones con los clientes, capacidad de innovación y cultura, por lo que una vez más, el capital humano puede considerarse uno de los activos más valiosos y según Sánchez (2014), la capacidad para trabajar en equipo e interactuar con otras personas de forma complementaria, coordinada, comunicativa, confiada y comprometida, es lo que permite la consecución de los objetivos institucionales.

Es claro que por mejores talentos que lleguen a la organización, si el ambiente laboral no brinda las condiciones necesarias para que el personal desarrolle todo su potencial, difícilmente se puede esperar que los resultados sean óptimos. Los stakeholders deben contar con un conjunto de características propias del entorno de trabajo que Hackman y Oldman (1980), referidos por Molero, Lois, García y Gómez (2017), han propuesto como condicionantes para un grupo se sienta motivado y son las siguientes: (1) que la labor a desarrollar requiera habilidades diferentes aportadas por distintos miembros del equipo, (2) que se produzcan resultados positivos tanto para el grupo como para sus miembros, (3) que los individuos puedan tener la suficiente autonomía como para realizar las actividades y (4) que puedan recibir feedback sobre cómo están haciendo su tarea.

\section{Conclusiones}

Existen diferentes conceptos que hacen referencia a la competitividad pero en un sentido más amplio, completo y acorde a las teorías de Rubio y Baz (2015) y Hernández (2000), se puede definir como un conjunto de factores que forman parte de las estructuras internas propias 
de la organización y que contribuyen a mejorar la posición que ocupa un producto o empresa en el mercado frente a otros productos fabricados por los competidores.

La competitividad constituye el motor impulsor del crecimiento y desarrollo para las organizaciones modernas; es por eso que actualmente las estrategias planteadas por la cúpula empresarial están direccionadas a la producción de bienes o servicios con valor agregado para el cliente con el fin de generar una ventaja competitiva que según los aportes de Porter (1980), permiten vencer a los competidores del mercado e implica un factor determinante para el éxito o fracaso de la compañía.

El liderazgo empresarial no se enfoca en el bienestar de la organización solamente sino también con el desarrollo personal y profesional de los stakeholders. El liderazgo conlleva una serie de cualidades propias tales como el carisma, empatía, capacidad de motivación y confianza necesarias para influir en las actitudes de otras personas en busca de mejorar su rendimiento y que esto se traduzca en beneficios para la organización; sin embargo, según Abarca (2013), no es una cualidad innata de cada persona, por ende, cualquier persona puede ser capaz de desarrollar, emplear los atributos del liderazgo y ponerlos en práctica de manera apropiada y con un propósito.

La productividad y la competitividad son dos términos estrechamente relacionados y ambos representan un emblema de vida institucional para las organizaciones modernas. Generalmente, se asume que si una industria es competitiva automáticamente es productiva, pero la competitividad según Huertas y Dominguez (2008), hace referencia a la calidad de los bienes o servicios entregados al mercado mientras que la productividad comprende el uso eficiente de recursos que contribuyen responder con rapidez los requerimientos del mercado. (Porter, 2017).

Son diversas las fuerzas que pueden afectar la competitividad de las organizaciones, la naturaleza del entorno empresarial es dinámica y requiere considerar factores internos y externos que pueden modificar las condiciones de la industria. La estrategia empresarial debe ser diseñada para facilitar la toma de decisiones acorde a los aportes de Carrión (2007) y tiene como finalidad ganar posicionamiento en el mercado y conseguir posiciones competitivas.

La globalización comprende el resultado de la integración de la economía a nivel mundial según Bueno y Morcillo (2014), esta situación que envuelve naturalmente al entorno empresarial, ha provocado que los productos o servicios puedan ser accesibles a clientes ajenos al país donde opera la empresa y por tanto, fabricar productos de calidad y con valor agregado percibido por el consumidor es objetivo que persiguen las empresas modernas en busca de abrir sus horizontes y captar nuevos clientes.

El capital humano según Blanco (2015), constituye uno de los activos intangibles mejor valorados por las organizaciones para desarrollar ventajas competitivas sostenibles en el tiempo. Actualmente existe una constante rivalidad entre las empresas por incorporar al equipo de trabajo, personal mejor entrenado y con experticias en diferentes áreas del conocimiento pues, esto permite combinar las capacidades y fortalezas de cada persona en pro de consolidar equipos de trabajo multidisciplinarios cuyo aporte en conjunto coadyuve al logro de los objetivos empresariales. 


\section{Referencias Bibliográficas}

Abarca, N. (2013). El líder como coach. Chile: El Mercurio Aguilar.

Alonso, V. (2010). Factores criticos de éxito y evaluación de la competitividad de destinos turísticos. Centro de Investigaciones y Estudios Turísticos. Argentina. Estudios y Perspectivas en Turismo, vol. 19, núm. 2, marzo, 2010, 201-220.

Anderson, P. (1982). Marketing, Strategic Planning and the Theory of the firm. Journal of Marketing, Vol. 46, $N^{\circ}$ 2, 15-26.

Barceló, M., Baglietto, A., Ballesteros, A., Correas, J., Fernández, P., Gómez, S Hernández, E., López, D; Moyano, J. (2001). Hacia una economía del conocimiento. Madrid: ESIC Editorial.

Blanco, C. (2015). Evidencias de la gestión de conocimiento en contextos sociales y tecnológicos de países de Latinoamérica y Europa. Bogotá: Omnia Publisher SL.

Bueno, E., Morcillo, P. (1994). Fundamentos de Economía y Organización Industrial. Madrid: McGraw-Hill.

Carrión, J. (2007). Estrategia. De la visión a la acción. . Madrid: ESIC.

Chiavenato, I. (1999). Introducción a la teoría general de la administración. Una ventaja competitiva. Colombia: McGraw-Hill.

Cordero, P., Chavarría, H., Echeverri, R., Sepúlveda, S. (2003). Territorios rurales, competitividad y desarrollo. San José: IICA.

Corredor, A. (2017). Desarrollo de proyectos para emprendedores. Madrid: RA-MA Editorial.

Cuatrecasas, L. (2016). Claves del lean management en tiempos de maxima competitividad. España: Profit.

Fernández, E., Montes, J., Vázquez, C. (1997). La competitividad de la empresa, un enfoque basado en la teoría de recursos. . Madrid: Universidad de Oviedo.

Fúquene, C. (2007). Producción limpia, contaminación y gestión ambiental. Bogotá: Pontificia Universidad Javeriana.

Galindo, M. (2008). Diccionario de Economía Aplicada: Política económica, economía mundial y extructura económica. Madrid: Ecobook.

Hernández, E. (2000). La competitividad industrial en México. México D.F: Plaza y Valdes Editores.

Huertas, R., Dominguez, R. (2008). Decisiones estratégicas para la direccion de operaciones en empresas de servicios y turísticas. Barcelona: Publicacions y edicions de la Universitat de Barcelona.

Kaplan, R., Norton, D. (2014). Mapas estratégicos. Barcelona: Gestión 2000.

Kotler, P. (1992). Dirección de Marketing. Madrid: Prentice Hall.

Kotler, P., Lane, K. (2006). Dirección de marketing. México D.F: Pearson.

López, M., Grandío, A. (2005). Capital Humano como fuente de ventajas competitivas. Madrid: Netbiblo.

Maxwell, J. (2004). El ABC del liderazgo. Buenos Aires: V\&R Editoras.

Miles, R., Snow, C. (1978). Organizational strategy, structure and process. New York: McGrawHill. 
Miller, D. (1987). Configurations of Strategy and Structure: Towards a Synthesis. Strategic Management Journal, vol. 7, p.223-249.

Mitzberg, H. (1988). Generic Strategies: Toward a Comprehensive Framework. Advances in Strategic Management, vol.5, JAI Press Inc. Greenwich, p.1-67.

Molero, F., Lois, D., García-Ael, C., Gómez, A. (2017). Psicología de los grupos. Madrid: UNED.

Olavarrieta de la Torre, J. (1999). Conceptos generales de productividad, sistemas, normalización y competitividad para la pequeña y mediana empresa. México D.F: Universidad Iberoamericana.

Porter, M. (1980). Competitive strategy. Techniques for analyzing industries and competitors. New York: The Free Press.

Porter, M. (1985). Estrategia Competitiva: Técnicas para el análisis de los sectores industriales y de la competencia. México D.F: Continental.

Porter, M. (2015). Estrategia competitiva. Técnicas para el análisis de los sectores industriales y de la competencia. México, D.F: Continental.

Porter, M. (2017). Ser competitivo. México D.F: Grupo Editorial Patria.

PSICORE. (2014). Perfil de liderazgo empresarial. Guatemala: PSICORE Ediciones.

Rojas, P., Chavarría, H., Sepúlveda, S. (1999). La competitividad en la agricultura: cadenas agroalimentarias y el impacto del factor localización espacial. San José: IICA.

Rubio, L., Baz, V. (2015). El poder de la competitividad. México D.F: DIDAC.

Sánchez, D. (2017). El mentor. Guía de mentoring para la gestión y el liderazgo empresarial. España: Almuzara.

Sánchez, M. (2014). Comunicación efectiva y trabajo en equipo. . Madrid: Editorial CEP. 